\section{TERMOGRAFIA MÉDICA INFRAVERMELHA APLICADA À MEDICINA DO ESPORTE}

\author{
APPLICATION OF MEDICAL INFRARED THERMOGRAPHY TO SPORTS MEDICINE
}

TERMOGRAFÍA MÉDICA INFRARROJA APLICADA A LA MEDICINA DEPORTIVA

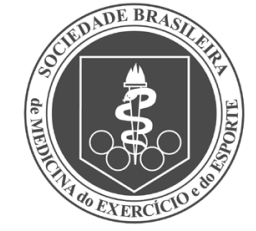

Artigo de ReVISÃo

REVIEW ARTICLES

ARTÍCULOS DE REVISIÓN
Ana Carolina Ramos e Côrte (Médica do Esporte)

Arnaldo José Hernandez (Médico do Esporte)

1. Universidade de São Paulo, Faculdade de Medicina, Instituto de Ortopedia e Traumatologia, Grupo de Medicina do Esporte, São Paulo, SP, Brasil.

2. Universidade de São Paulo, Faculdade de Medicina, Departamento de Ortopedia e Traumatologia, Grupo de Medicina do Esporte, São Paulo, SP, Brasil.

\section{Correspondência:}

Rua Dr. Ovídio Pires de Campos, 333, Cerqueira Cesar, São Paulo, SP, Brasil. 05403-010.

anacarolinacorte@usp.br

\begin{abstract}
RESUMO
A termografia médica infravermelha é um instrumento de análise não invasiva e não radioativa, capaz de analisar funções fisiológicas relacionadas com o controle da temperatura da pele. A termografia detecta a luz infravermelha emitida pelo corpo e visualiza mudanças de temperatura corporal relacionadas à alteração no fluxo sanguíneo. Não é um método que mostra anormalidades anatômicas, porém é capaz de mostrar mudanças fisiológicas. Existem várias aplicações da termografia no campo da medicina: desordens neurológicas, reumatológicas, musculares, doenças vasculares, patologias urológicas, ginecológicas, ortopédicas e na medicina esportiva. Para todas as áreas médicas, a termografia está estabelecida como uma medida que proporciona um mapeamento visual da distribuição da temperatura da pele. A termografia não deve ser usada como ferramenta diagnóstica única. Exames clínicos devem ser realizados para interpretação dos termogramas. Nas aplicações médicas, esta técnica proporciona, somente, uma imagem da distribuição da temperatura da pele; não é capaz de mostrar dados de uma superfície profunda do corpo, como é possível por outros exames de imagem. Entretanto, é um método não-invasivo e objetivo, além de seguro e inofensivo. Na medicina do esporte, o uso da termografia pode proporcionar melhores resultados aos atletas por ser um instrumento na identificação de riscos e na prevenção de lesões, além de ser uma importante ferramenta no acompanhamento do treinamento esportivo, a partir da avaliação da quantificação da carga de trabalho.
\end{abstract}

Descritores: raios infravermelhos; reprodutibilidade dos testes; medicina esportiva/métodos; termografia/métodos.

\begin{abstract}
Medical infrared thermography is a non-invasive, non-radioactive detection tool that is capable of analyzing physiological functions related to skin temperature control. Thermography detects infrared light emitted by the body and visualizes changes in body temperature related to changes in blood flow. It is not a method that shows anatomical abnormalities, but it is capable of showing physiological changes. There are various applications of thermography in the field of medicine: neurological, rheumatological and muscular disorders, vascular diseases, urological, gynecological and orthopedic disorders, and sports medicine. For all medical fields, thermography is established as a measure that provides a visual map of skin temperature distribution. Termography should not be used as the sole diagnostic tool. Clinical examinations should be performed to interpret the thermograms. In medical applications, this technique only provides an image of the distribution of skin temperature; it is not capable of showing data for a deep surface layers of the body, as is possible with other imaging exams. However it is a non-invasive, objective method, as well as being safe and harmless. In sports medicine, the use of thermography can provide better results for athletes, as it is an instrument for identifying risks and preventing injuries, as well as an important tool for monitoring sports training, based on the evaluation of training load.
\end{abstract}

Keywords: infrared rays; reproducibility of results; sports medicine/methods; thermography/methods.

\section{RESUMEN}

La termografía médica infrarroja es un instrumento para el análisis no invasivo y no radiactivo, capaz de analizar las funciones fisiológicas relacionadas con el control de temperatura de la piel. La termografía detecta la luz infrarroja emitida por el cuerpo y muestra los cambios relacionados con la temperatura corporal con respecto a la variación del flujo sanguíneo. No es un método que muestra anormalidades anatómicas, pero es capaz de mostrar los cambios fisiológicos. Hay varias aplicaciones de la termografía en la medicina: trastornos neurológicos, reumáticos, musculares, enfermedades vasculares, enfermedades urológicas, ginecológicas, ortopédicas y en la medicina deportiva. Para todos los campos de la medicina, la termografía se establece como una medida que proporciona un mapa visual de la distribución de la temperatura de la piel. La termografía no debe utilizarse como la única herramienta de diagnóstico. El examen clínico debe llevarse a cabo para la interpretación de los termogramas. En aplicaciones médicas, esta técnica sólo proporciona una imagen de la distribución de la temperatura de la piel, no siendo posible mostrar datos de las superficies más profundas del cuerpo, al igual que con otros exámenes por imágenes. Sin embargo, es un método no-invasivo y objetivo, así como seguro e inocuo. En la medicina deportiva, el uso de la termografía puede proporcionar mejores resultados para los atletas ya que es un instrumento para la identificación de riesgos y la prevención de lesiones, además de ser una herramienta importante en el control del entrenamiento deportivo, basado en la evaluación de la cuantificación de la carga trabajo.

Descriptores: rayos infrarrojos; reproducibilidad de resultados; medicina deportiva/métodos; termografía/métodos. 


\section{INTRODUÇÃO}

Como ciência médica, a termologia foi, primeiramente, documentada em 400 a.C. por Hipócrates. Nesta época, Hipócrates desenvolveu a teoria de que "Em qualquer parte do corpo, se houver excesso de calor ou de frio, a doença existe e é pra ser descoberta."

A partir dessa teoria, a ciência evoluiu e o cientista William Hershell, no ano de 1800, descobriu a mensuração da temperatura em cada cor do arco-íris, compreendendo, assim, o espectro de cores. William Hershell descobriu o que hoje chamamos de infravermelho. Infravermelho, que significa "abaixo do vermelho", descreve uma extensão específica do espectro electromagnético, uma escala usada para classificar várias formas de emissão de energia.

Além dos conhecimentos de William Hershell, a teoria de Albert Einstein sobre o efeito fotoelétrico foi fundamental para expandir a compreensão dos fundamentos físicos da energia infravermelha. Em 1950, o exército americano aplicou os princípios da termologia no desenvolvimento de projetos de defesa militar, na tentativa de capturar informações de temperatura, usando câmeras de imagem infravermelha. Estes dispositivos modernos revolucionaram a ciência e rapidamente substituíram muitas das formas mais primitivas de medição de temperatura, tais como termômetros de contato e termometria de cristal líquido. A vantagem destes novos dispositivos de imagem é fornecer uma maneira de capturar instantaneamente imagens termográficas. A partir desses estudos, surgiu a termografia médica infravermelha'.

A qualidade das imagens e a falta de padrões metodológicos, no passado, limitaram a veracidade dos estudos, resultando em uma técnica não aceita ${ }^{2}$. Com o avanço tecnológico das câmeras infravermelhas nos últimos anos, a termografia tornou-se um instrumento de medida eficaz no diagnóstico de patologias ${ }^{3}$. Em 1987, a American Medical Association reconheceu a termografia infravermelha como um instrumento diagnóstico factível.

\section{Termografia médica}

Como efeito natural de metabolismo, o ser humano libera constantemente diferentes níveis de energia no comprimento de onda infravermelho, e esta informação pode ser expressa e medida na forma de calor.

A termografia médica infravermelha é um instrumento de análise não invasiva e não radioativa capaz de analisar funções fisiológicas relacionadas ao controle da temperatura da pele, importante órgão na regulação da temperatura corporal ${ }^{4}$. A termografia detecta a luz infravermelha emitida pelo corpo e visualiza mudanças na temperatura corporal relacionadas à alteração no fluxo sanguíneo ${ }^{5}$. Não é um método que mostra anormalidades anatômicas, porém é capaz de mostrar mudanças fisiológicas ${ }^{6}$.

A maioria dos instrumentos diagnósticos por imagem utiliza porções do espectro eletromagnético ${ }^{7}$ (Figura 1). Entretanto, diferentemente de outros dispositivos médicos, a termografia é um instrumento não radioativo, permitindo, então, aplicações irrestritas e com segurança.

Para realização de uma análise termográfica é importante conhecer fatores que influenciam o resultado do exame. Dentre eles estão presentes: fatores ambientais (tamanho da sala de coleta, temperatura ambiental, umidade relativa do ar, pressão atmosférica e radiação), fatores técnicos (camera, protocolo, software, análise estatística) e fatores individuais (sexo, idade, antropometria, ritmo circadiano, emissividade da pele, uso de medicamentos e prática de exercício físico). Porém, se controlados, não há prejuízo no resultado final ${ }^{8}$.

\section{Termorregulação}

O ser humano é capaz de manter uma temperatura corporal constante, independente do meio ambiente. E esta temperatura é

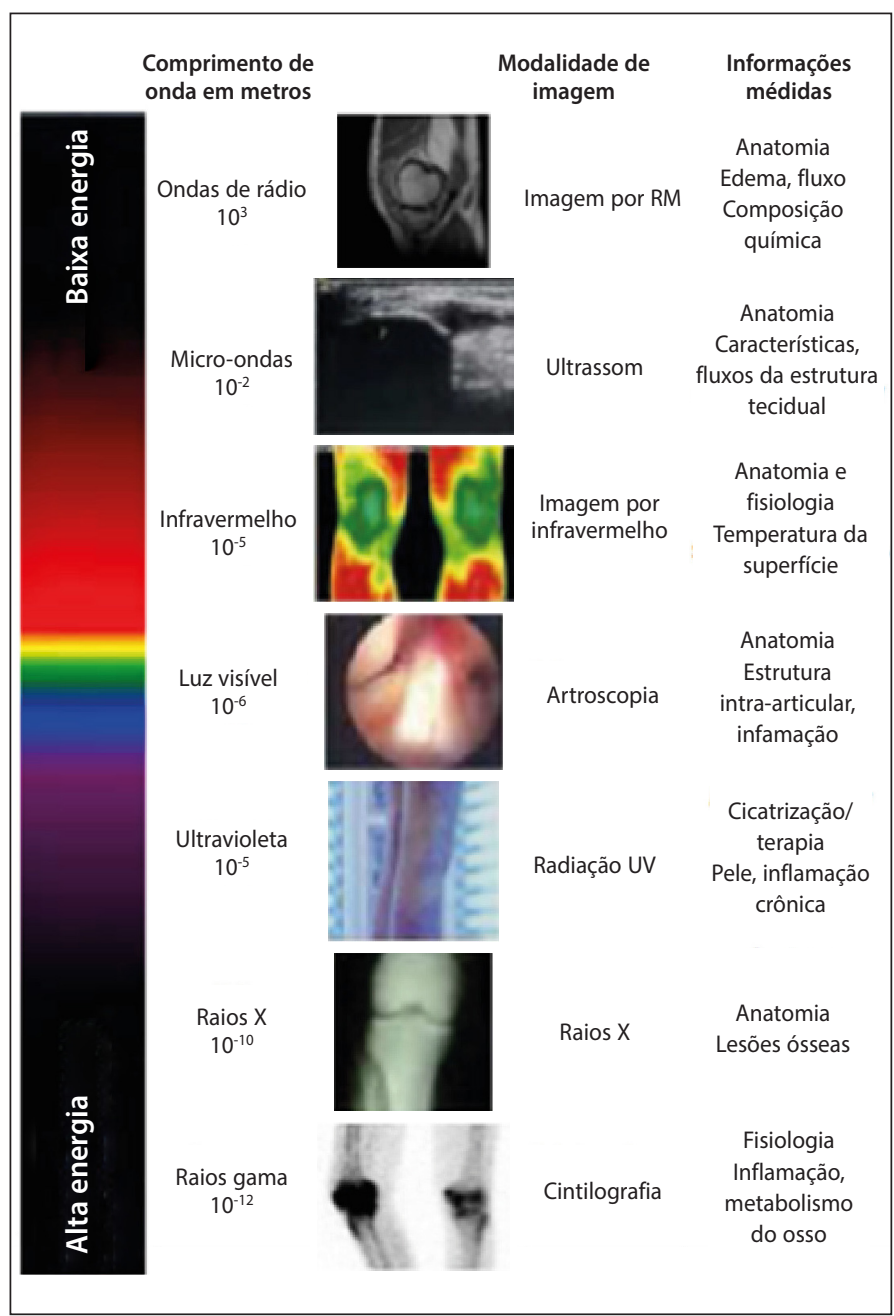

Figura 1. Exames diagnósticos por imagem com espectro eletromagnético.

preservada num limite estreito de 33-42 graus celsius. Mudança dessa temperatura é considerada, claramente, um indicador de doença. E o controle da temperatura corporal se dá por um processo fisiológico chamado termorregulação ${ }^{9}$.

A física da radiação de calor e a fisiologia da termorregulação humana trazem a segurança e a validação da interpretação de imagens térmicas. A regulação da temperatura da pele é um sistema complexo que depende da taxa de fluxo sanguíneo, das estruturas do tecido subcutâneo e da atividade do Sistema Nervoso Simpático (SNS)9 ${ }^{9}$. Entretanto, há evidências que o SNS é o regulador primário da circulação sanguínea na pele, sendo, portanto, o principal regulador da emissão térmica ${ }^{10}$.

Termorreceptores da pele, também conhecidos como Corpúsculos de Ruffini, reconhecem a temperatura ambiental. Um aumento da temperatura resulta na vasodilatação, levando ao aumento do fluxo sanguíneo; enquanto a vasoconstrição ocorre pela diminuição da temperatura e resulta na redução do fluxo sanguíneo da pele. Esse processo fisiológico é associado à transferência de calor por métodos como convecção, condução, radiação e evaporação. Portanto, o mecanismo de termorregulação é complexo e ainda não muito bem compreendido ${ }^{11}$.

A associação entre temperatura corporal humana e doença é tão antiga quanto a medicina. As imagens térmicas têm sido usadas para o estudo de várias doenças em que a temperatura da pele pode refletir a presença de inflamação tecidual, ou onde há alteração de fluxo sanguíneo devido à anormalidade clínica. Em princípio, a imagem térmica pode ser aplicada na medicina como diagnóstico e acompanhamento evolutivo da patologia ${ }^{6}$.

Existem várias aplicações da termografia no campo da medicina. 
São elas: desordens neurológicas, reumatológicas, musculares, dermatológicas, doenças vasculares, patologias urológicas, ginecológicas, ortopédicas e na Medicina Esportiva ${ }^{7}$. Para todas as áreas médicas, está estabelecida a termografia como uma medida que proporciona um mapeamento visual da distribuição da temperatura da pele, mas não quantifica valores absolutos de temperatura. Além disso, a termografia não deve ser usada como ferramenta diagnóstica única. Exames clínicos devem ser realizados para interpretação dos termogramas. Nas aplicações médicas, esta técnica proporciona, somente, uma imagem da distribuição da temperatura da pele; não é capaz de mostrar dados de uma superfície profunda do corpo, como é possível por outros exames de imagem. Entretanto, é um método não-invasivo e objetivo, além de seguro e inofensivo. Várias instituições médicas mundiais entendem a necessidade de publicações de trabalhos científicos para consolidar a aplicação prática da termografia na medicina ${ }^{6}$

\section{Artrite reumatóide}

Recentemente, um estudo piloto mostrou uma forte correlação entre alta temperatura e presença de edema em articulações de dedos, em pacientes com artrite reumatóide. Quando uma articulação está inflamada em fase aguda, o aumento de calor pode ser detectado pelo toque. Entretanto, discretas mudanças na temperatura da superfície articular representam redução ou exacerbação da inflamação. A termografia é, portanto, capaz de detectar precocemente essas mudanças, conduzindo a introdução do tratamento; seja farmacológico, físico ou cirúrgico ${ }^{12}$. Osteoatrite: estudos mostram uma boa correlação entre aumento de temperatura e alterações radiográficas importantes em osteoartrite de joelho ${ }^{13,14}$. Circulação periférica: em alguns distúrbios vasculares, como fenômeno de Raynaud, os danos causados pela vasoconstrição periférica podem ser visualizados na termografia ${ }^{15}$.

Câncer de mama: tumores são caracterizados pelo aumento da angiogênese levando ao aumento na atividade metabólica e da temperatura local comparada ao tecido adjacente. A partir, desse dado, a técnica da termografia ganhou grande espaço no diagnóstico e evolução do câncer de mama ${ }^{16}$.

Melanoma: estudos mostram importante relação entre o diagnóstico de melanoma e a alterações no padrão térmico da pele ${ }^{17,18}$

Neuropatias: a análise termografia tem se tornado uma grande ferramenta no monitoramento de neuropatias, entre elas: Síndrome dolorosa regional e acidente vascular cerebral ${ }^{19}$

\section{Aplicação na medicina do esporte}

Na Medicina do Esporte, o uso da termografia pode proporcionar melhores resultados aos atletas por ser um instrumento na identificação de riscos e na prevenção de lesões, além de ser uma importante ferramenta no acompanhamento do treinamento esportivo, a partir da avaliação da quantificação da carga de trabalho ${ }^{20-22}$. Os atletas são expostos a um estresse físico do treinamento e de competições. Reações por sobrecarga são frequentes; portanto, diagnósticos precoces são importantes. Além do diagnóstico precoce, a localização da inflamação é um passo fundamental no tratamento correto. A termografia também possibilita monitorar a temperatura da superfície corporal antes, durante e após o movimento e detectar mudanças na temperatura da pele causadas pelo exercício ${ }^{4,23-25}$.

A circulação periférica tem uma importante função no tratamento de lesões e na termorregulação. Para diferenciar as mudanças de temperaturas por lesões das mudanças de temperatura pela sobrecarga de exercício, é preciso compreender as diferentes respostas fisiológicas das estruturas envolvidas ${ }^{26}$.

Para definir se um termograma está normal, uma pesquisa da University of Glamorgan criou uma base de dados de imagens térmicas de diferentes partes do corpo de indivíduos saudáveis. A simetria entre membros é essencial na avaliação da normalidade do termograma (Figura 2).

A literatura tem mostrado que uma diferença maior que 1 grau centígrado entre os lados do corpo pode indicar um processo patofisiológico ${ }^{27}$ (Figura 3).

Já Carmona, em sua tese de doutorado, iniciou o trabalho de prevenção de lesão em jogadores de futebol da Liga Espanhola, no ano de 2009, a partir do resultado da análise termográfica. Com uma assimetria maior que 0,3 graus centígrados, os jogadores foram submetidos a protocolos de prevenção. Os resultados mostraram uma redução significativa de $60 \%$ na incidência de lesões durante a pré-temporada de 2009 em relação à pré-temporada de $2008^{21}$ (Figura 4, Tabela 1).

Uma lesão causa alteração do fluxo sanguíneo que, por sua vez, afeta a temperatura da pele. Várias condições estão associadas a vasodilatação ou vasoconstrição regional, hiperperfusão, hipervascularização e hipermetabolismo que levam a altas temperaturas na superfície da pele. Médicos

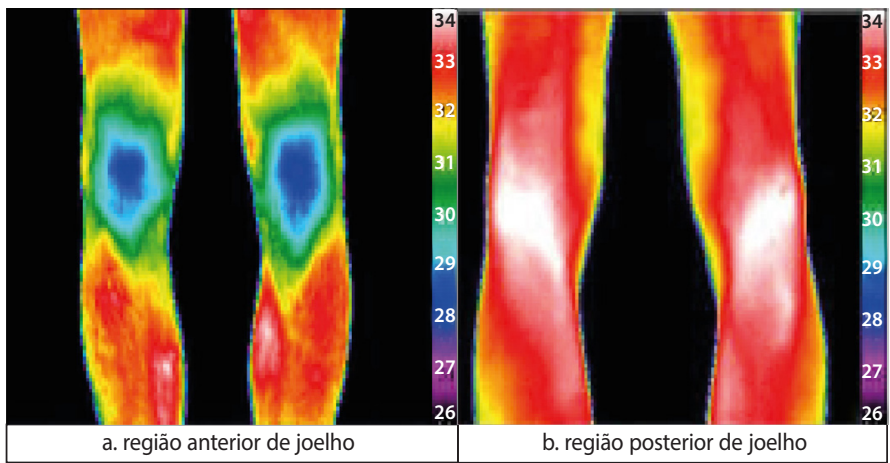

Figura 2. Simetria termográfica em joelhos saudáveis.

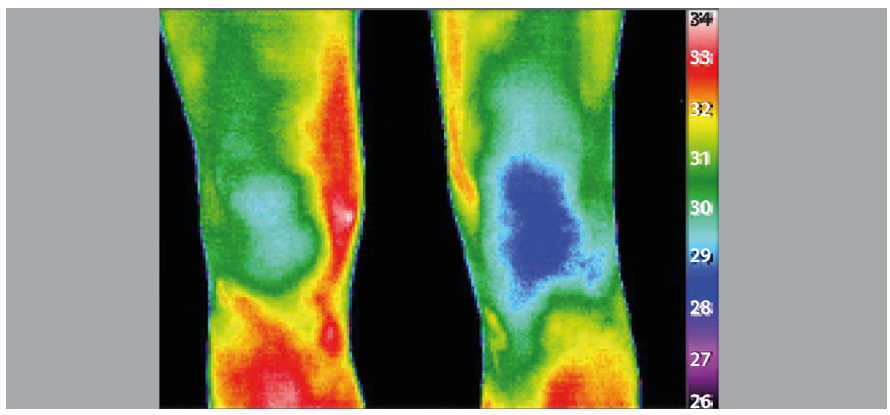

Figura 3. Imagem termográfica de condropatia patelar grau III em joelho esquerdo.

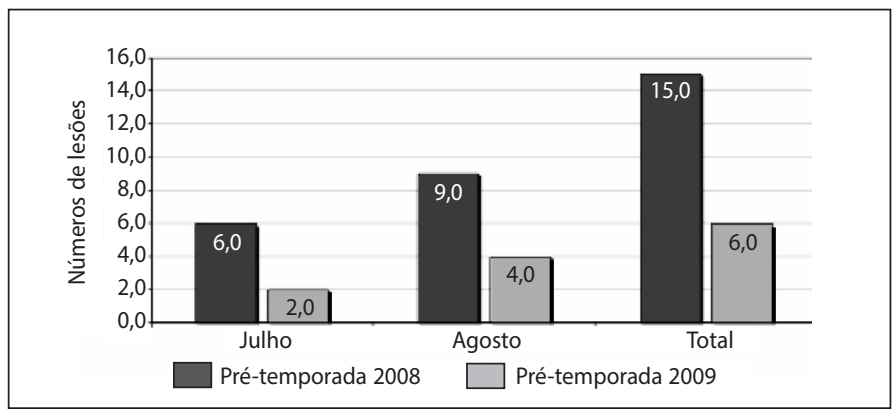

Figura 4. Número de lesões por meses nas pré-temporadas de 2008 e 2009.

Tabela 1. Percentural de jogadores que sofreram uma lesão ao longo da pré-temporada de 2008 e 2009

\begin{tabular}{c|c|c}
\hline Pré-temporada & \% Lesão & \% Sem lesão \\
\hline 2008 & $45,8 \%$ & $54,2 \%$ \\
\hline 2009 & $20,8 \%$ & $79,2 \%$ \\
\hline Total & $33,3 \%$ & $66,6 \%$ \\
\hline
\end{tabular}


necessitam de conhecimento específico dos sinais térmicos fisiológicos e dos sinais térmicos patológicos específicos do esporte para uma intervenção precoce e segura. Além disso, a evolução do tratamento de lesões pode ser monitorado utilizando imagens térmicas. Entretanto, isso requer comparação de imagens basais para seguimento da lesão ${ }^{26}$.

Em relação a lesões por sobrecarga, imagens periódicas esclarecerão se a distribuição assimétrica da temperatura é preditiva de reação por sobrecarga sintomática. Um relato de caso mostrou o termograma de um jogador de futebol de 18 anos em repouso com padrões simétricos. Após uma sessão de treino, houve diferença visível no termograma dos joelhos. O atleta não relatou dor no momento (Figura 5). Entretanto, durante a temporada, relatou uma dor carga-dependente, difusa, em joelho esquerdo. O exame clínico confirmou a dor à palpação em região medial de joelho sem outras manobras positivas. O termograma mostrou um local de reação por sobrecarga e, a partir de então, o atleta foi orientado a reduzir o estresse físico excessivo ${ }^{28}$.

Epidemiologicamente, estudos mostram uma alta incidência de lesões em pés, joelhos, tornozelos em corredores recreativos e competitivos $^{29}$. A maiora das lesões em atletas recreativos são traumáticas; porém, em atletas com volume semanal alto de treinamento, são as lesões por sobrecarga que se destacam. Entre elas: fratura por estresse, tendinite patelar, síndrome do estresse tibial, e mais prevalentemente tendinite de calcâneo. Em outro relato de caso, um atleta de 22 anos, que corre cerca de 40-100 km por semana, apresentou diagnóstico de tendinopatia da porção medial do calcâneo direito (Figura 6). A imagem termográfica mostrou uma temperatura de 1,6 graus abaixo do lado contralateral saudável. A baixa temperatura pode indicar baixa atividade metabólica pela perda da estrutura normal das fibras ${ }^{28}$.

Após oito semanas e meia de tratamento foram realizadas duas imagens termográficas: repouso e após 45 min de corrida de baixa intensidade. No momento pré-exercício a diferença reduziu para 0,6 graus e após o exercício, diferença reduziu para 1 grau $^{28}$ (Figura 7).

As lesões traumáticas envolvem um longo e dispendioso período de reabilitação. Alta qualidade no tratamento pode reduzir o tempo de retorno ao esporte. Sabe-se que quanto mais vascularizada for a

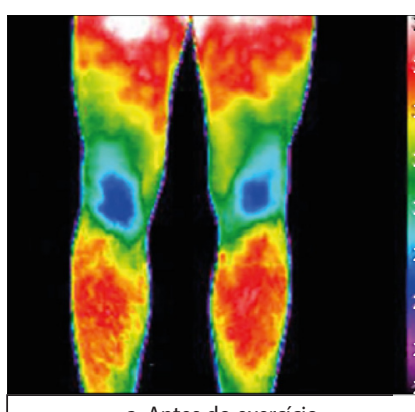

a. Antes do exercício

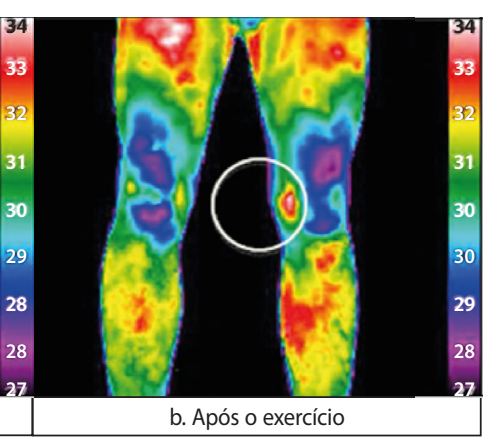

Figura 5. Imagem termográfica mostrando aumento da temperatura em região medial de joelho esquerdo.

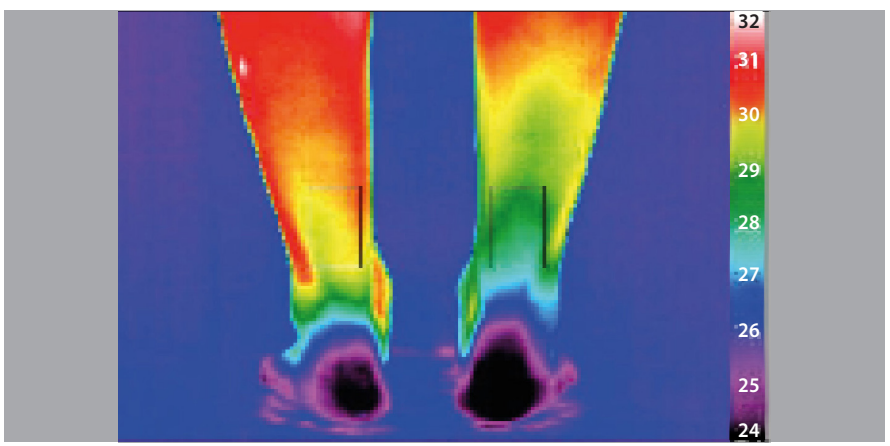

Figura 6. Tendinopatia na porção medial do calcâneo direito. área lesionada, mais rápida é a cura comparada com áreas pouco vascularizadas $^{30}$. A termografia, então, pode trazer informações sobre o estado de vascularização e a evolução do tratamento.

A maioria das análises de energia do corpo humano submetido ao exercício físico é baseada na avaliação do trabalho executado e a sua eficiência ${ }^{31}$. Portanto, a termografia, além da aplicação médica, pode servir como um instrumento importante no acompanhamento do treinamento esportivo, por possibilitar a verificação da região corporal com maior gasto energético após a execução de um exercício ${ }^{32}$.

Arfaoui et al..$^{32}$ estudaram o resultado da temperatura corporal após um atleta realizar quatro tiros de 100 metros, sendo cada tiro de um estilo diferente (borboleta, costas, peito e crawl). Com os resultados, conseguiu verificar a região corporal com maior temperatura após cada tiro $^{2}$. A Figura 8 mosta o resultado termografico do atleta.

A Tabela 2 mostra a média da temperatura corporal do atleta após os 4 estilos.

Observa-se, portanto, que a maior temperatura corporal, para o atleta, corresponde ao nado de costas. Este resultado confirmou dados

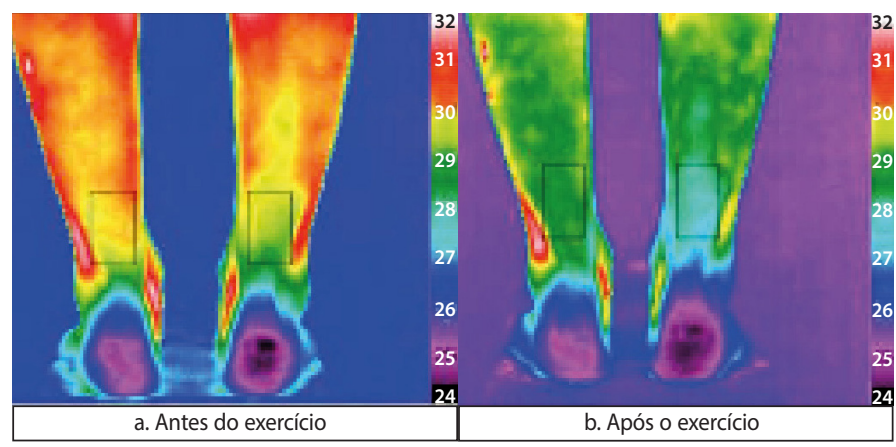

Figura 7. Imagem termográfica de tendão.

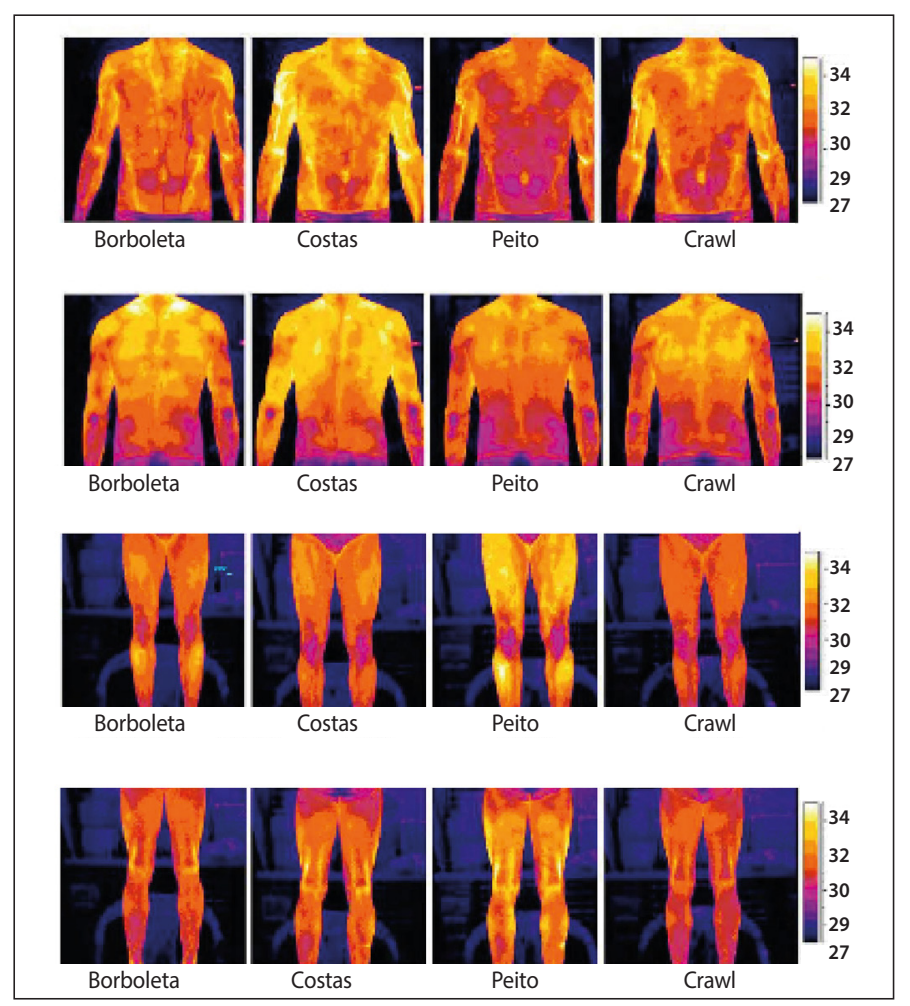

Figura 8. Imagens termográficas após cada estilo de nado.

Tabela 2. Valores médios da temperatura cutânea em cada estilo de nado ${ }^{32}$

\begin{tabular}{c|c|c|c|c}
\hline \multirow{2}{*}{ Média } & Borboleta & Costas & Peito & Crawl \\
\cline { 2 - 5 } & 31,73 & 32,14 & 31,42 & 31,58 \\
\hline
\end{tabular}


prévios da temperatura que também mostraram o nado de costas como o nado de maior gasto energético e confirmou a possibilidade de verificação, a partir da termografia, da região corporal com maior gasto energético após a execução de um exercício ${ }^{8,32}$.

\section{CONSIDERAÇÕES FINAIS}

A termografia parece ser bastante útil por ser um método confiável, não invasivo e bastante seguro. Sua grande utilidade parece ser na triagem de atletas para lesões por sobrecarga sendo o principal desafio combinar as informações anatômicas e fisiológicas demonstrado pelo padrão térmico da pele. Além disso tem um importante papel na quantificação da carga de treino.

O rastreio térmico de atletas lesionados e não lesados é o primeiro passo para criar um banco de dados específico do esporte com termo- gramas individuais. Medidas de acompanhamento repetida durante a temporada de esporte irão esclarecer a ligação entre as distribuições de temperatura assimétricas, alterações fisiopatológicas na superfície da pele e a extensão da lesão.

A principal vantagem da termografia é a sua segurança, contudo, sua desvantagem resulta das suas limitações físicas. A técnica bidimensional e não radiante fornece informações sobre estruturas superficiais.

Deve-ser lembrar que o uso da termografia na medicina do esporte não é substituir o exame clínico, porém complementar a avaliação e dar suporte às decisões.

Todos os autores declararam não haver qualquer potencial conflito de interesses referente a este artigo.

\section{REFERÊNCIAS}

1. Rogalski A. Recent progress in infrared detector technologies. Infrared Phys Technol. 2011;54:136-54.

2. Elliot RL, Head JF. Medical infrared imaging in the twenty-first century. Thermol Int. 1999;9(4):111.

3. Diakides NA, Bronzino JD, editors. Medical infrared imaging. Boca Raton: CRC Press; 2008.

4. Merla A, Mattei PA, Di Donato L, Romani GL. Thermal imaging of cutaneous temperature modifications in runners during graded exercise. Ann Biomed Eng. 2010;38(1):158-63.

5. Melnizky P, Schartelmüller T, Ammer K. Prüfung der intra- und interindividuellen Verlässlichkeit der Auswertung von Infrarot-Thermogrammen. Eur J Thermol. 1997;7: 224-6.

6. Ring EF, Ammer K. Infrared thermal imaging in medicine. Physiol Meas. 2012;33(3):R33-46.

7. Hildebrandt C, Raschner C, Ammer K. An overview of recent application of medical infrared thermography in sports medicine in Austria. Sensors (Basel).2010;10(5):4700-15.

8. Priego Quesada JI, Carpes FP, Bini RR, Salvador Palmer R, Pérez-Soriano P, Cibrián Ortiz de Anda RM. Relationship between skin temperature and muscle activation during incremental cycle exercise. J Therm Biol. 2015;48:28-35.

9. Kellog DL, Pérgola P. (2000). Skin Response to exercise and training. In: Garrett, WE, Kirkendall DT. Exercise and Sports Science. Philadelphia: Lippincott Williams \&Wilkins; 2000. p. 239-50.

10. Charkoudian N. Skin blood flow in adult human thermoregulation: how it works, when it does not, and why. Mayo Clin Proc. 2003;78(5):603-12.

11. Blatteis CM, editor. Physiology and pathophysiology of temperature regulation. Singapore: World Scientific Printers; 1998.

12. Spalding SJ, Kwoh CK, Boudreau R, Enama J, Lunich J, Huber D, et al. Three-dimensional and thermal surface imaging produces reliable measures of joint shape and temperature: a potential tool for quantifying arthritis. Arthritis Res Ther. 2008;10(1):R10.

13. Denoble AE, Hall N, Pieper CF, Kraus VB. Patellar skin surface temperature by thermography reflects knee osteoarthritis severity. Clin Med Insights Arthritis Musculoskelet Disord. 2010;3:69-75.

14. Warashina H, Hasegawa Y, Tsuchiya H, Kitamura S, Yamauchi KI, Torii Y, et al. Clinical, radiographic, and thermographic assessment of osteoarthritis in the knee joints. Ann Rheum Dis. $2002 ; 61(9): 852-4$

15. Ammer K. Cold challenge to provoke a vasospastic reaction in fingers determined by temperature measurements; a systematic review. Thermol Int. 2009;19(4):109-18.

16. Kontos M, Wilson R, Fentiman I. Digital infrared thermal imaging (DITI) of breast lesions: sensitivity and specificity of detection of primary breast cancers. Clin Radiol. 2011;66(6):536-9.

17. Çetingül MP, Herman C. Quantification of the thermal signature of a melanoma lesion. Int J Therm Sci. 2011;50:421-31.
18. Bonmarin M, Le Gal FA. Lock-in thermal imaging for the early-stage detection of cutaneous melanoma: a feasibility study. Comput Biol Med. 2014;47:36-43.

19. Neves EB, Vilaça-Alves J, Rosa C, Reis VM. Thermography in Neurologic Practice. Open Neurol J. 2015;9:24-7

20. Brukner P, Khan K. Brukner \& Khan's clinical sports medicine. 4th ed. Australia: McGraw-Hill Education; 2006.

21. Carmona PMG. Influencia de la información termográfica infrarroja en el protocolo de prevención de lesiones de un equipo de fútbol profesional español [tesis]. Madrid: Universidad Politécnica de Madrid, Facultad de Ciencias de la Actividad Física y del Deporte; 2012.

22. Neves EB, Reis VM. Fundamentos da termografia para o acompanhamento do treinamento desportivo. Rev Uniandrade. 2014;15(2):79-86.

23. Ferreira JJ, Mendonça LC, Nunes LA, Andrade Filho AC, Rebelatto JR, Salvini TF. Exercise-associated thermographic changes in young and elderly subjects. Ann Biomed Eng. 2008;36(8):1420-7.

24. Hardaker NJ, Moss AD, Richards J, Jarvis S, McEwan C, Selfe J. The relationship between skin surface temperature measured via non-contact thermal imaging and intra-muscular temperature of the rectus femoris muscle. Thermol Int. 2007;17(2):45-50.

25. Ring EF, Ammer K. Thermal imaging in sports medicine. Sport Med Today. 1998;1(2):108-9.

26. Mangine RE, Siqueland KA, Noyes FR. The use of thermography for the diagnosis and management of patellar tendinitis. J Orthop Sports Phys Ther. 1987;9(4):132-40.

27. Selfe J, Whitaker J, Hardaker N. A narrative literature review identifying the minimum clinically important difference for skin temperature asymmetry at the knee. Thermol Int. 2008;18(2):41-4.

28. Hidebrandt, C.; Zeilberger, K.; Ring, EFJ.; Raschner, C. The application of medical infrared thermography in Sports Medicine. Infrared Thermography. An International Perspective on Topics in Sports Medicine and Sports Injury, 2012, p. 257-74. Disponível em: http://www.infraroodscreening.com/publicaties/ InTech-The_application_of_medical_infrared_thermography_in_sports_medicine.pdf

29. Rosa BB, Asperti AM, Helito CP, Demange MK, Fernandes TL, Hernandez AJ. Epidemiology of sports injuries on collegiate athletes at a single center. Acta Ortop Bras. 2014;22(6):321-4.

30. Singer AJ, Clark RA. Cutaneous wound healing. N Engl J Med. 1999;341(10):738-46

31. Mady CE, Albuquerque C, Fernandes TL, Hernandez AJ, Saldiva PH, Yanagihara Jl, Oliveira Junior S. Exergy performance of human body under physical activities. Energy. 62;2013:370-8.

32. Arfaoui A, Polidori G, Taiar R, Popa C. Infrared thermography in sports activity. Infrared Thermography. An International Perspective on Topics in Sports Medicine and Sports Injury, 2012, p. 141-68. Disponível em: http://cdn.intechopen.com/pdfs/32034/InTech-Infrared_thermography_in_sports_activity.pdf 